\title{
Blood incubation infectivity test (BIIT): usefulness as compared to the SRA- PCR in the identification of Trypanosoma brucei rhodesiense in animal reservoirs
}

\author{
Charles WAISWA ${ }^{1 *}$, Kim PICOZZI ${ }^{2}$, William OLAHO-MUKANI ${ }^{3}$, \\ Eli KATUNGUKA-RWAKISHAYA ${ }^{1}$ and Susan C. WELBURN ${ }^{2}$ \\ ${ }^{1}$ Makerere University, Faculty of Veterinary Medicine, P.O. Box 7062, Kampala Uganda. \\ ${ }^{2}$ Centre for Tropical Veterinary Medicine, Royal (Dick) School Of Veterinary Medicine, University of \\ Edinburgh, EH25 9RG, Scotland, UK. \\ ${ }^{3}$ Directorate of Animal Resources, Ministry of Agriculture Animal Industry and Fisheries, P.O. Box 513, \\ Entebbe, Uganda. \\ *Corresponding author; E-mail: cwaiswa@vetmed.mak.ac.ug / cwaiswa@yahoo.co.uk
}

\begin{abstract}
This investigation is aimed at comparing the Blood Incubation Infectivity Test (BIIT) with the Serum Resistance Associated gene Polymerase Chain Reaction (SRA-PCR) techniques as tools that can be used to detect the presence of Trypanosoma brucei rhodesiense parasites in domestic animals reservoirs. Blood samples from 4754 animals (cattle, pigs and small ruminants) were screened for the presence of Trypanozoon parasites by microscopy and in total, 109 samples (98 T. bruce $i$ sl isolates from domestic animal and 11 known T. b. rhodesiense) were analysed. There was $81.6 \%$ agreement between the results from the BIIT and the SRAPCR, with Cohen Kappa of 0.63 suggesting a substantial agreement between the two tests. When SRA-PCR was taken as the reference standard, the BIIT test had a sensitivity of $82.1 \%$ and specificity of $81.4 \%$. The positive and negative predictive values were $74.4 \%$ and $87.3 \%$ respectively. Therefore, although the BIIT still remains a feasible test for detecting human infective trypanosomes in many African laboratories, SRA-PCR offers a quicker method for the identification of the animal reservoir for $T$. $b$. rhodesiense to enable Governments make firm decisions on public good investment on treating infected animals for the purpose of controlling the zoonotic sleeping sickness.
\end{abstract}

(C) 2010 International Formulae Group. All rights reserved.

Keywords: Trypanosoma brucei rhodesiense, zoonotic sleeping sickness, SRA-PCR techniques, domestic animals.

\section{INTRODUCTION}

Sleeping sickness is a disease caused by two parasites, Trypanosoma $b$. gambiense in Western and Central Africa and T. $b$. rhodesiense in Eastern Africa. The zoonotic nature of $T . \quad b$. rhodesiense results in a "hidden" reservoir of this parasite within the local animal population, thus maintaining the cycle of transmission of this parasites. The ability to differentiate $T$. b. rhodesiense from T. b. brucei has for many years been based on the parasites ability to survive in human serum. The link between human serum and the human infectivity of pathogenic 
trypanosomes was first realised in the early decades of the $20^{\text {th }}$ century (Fairbairn, 1933). The relationship between trypanosomal survival in human serum and $T . b$. rhodesiense led to the development of the Blood Incubation Infectivity Test (BIIT) (Rickman \& Robson, 1970a, 1970b), a simple but time consuming test for in vitro human serum resistance. The BIIT test capitalises on the only phenotypically distinguishing feature to differentiate between the co-existing human infective $T$. $b$. rhodesiense and the otherwise identical T. b. brucei within the animal population.

As molecular techniques evolved, the sensitivity of this technique was brought into doubt with a claim that human isolated trypanosomes show serum-sensitive (HSS) reactions on first testing with instances where the status of isolates change from sensitive to resistant forms (Rickman et al., 1991). The ability of $T$. b. rhodesiense to survive within human serum has been attributed to the role played by the Serum Resistant Associated protein (SRA) (Xong, 1998). The detection of this gene has since been developed as a molecular marker system to distinguish T.b. rhodesiense from other Trypanozoons by SRA-PCR (Welburn et al., 2001; Radwanska et al., 2002). However in terms of accessibility, the BIIT still remains the most feasible test for human infective trypanosomes in some African laboratories. This study was aimed at assessing its usefulness in being an indicator of the presence of the domestic animal reservoir using samples collected from south eastern Uganda, an area that had earlier been reported to have domestic animal reservoir for $T . b$. rhodesiense (Waiswa et al., 2003). Therefore, this paper presents data on the efficacy of the BIIT test in identifying potentially human infective parasites when compared to the SRA-PCR.

\section{MATERIALS AND METHODS Study area}

The study was based on trypanosome isolates collected from the endemic sleeping sickness foci of south eastern Uganda (Waiswa et al., 2003) where cattle have been identified as the main animal host for $T$. brucei sl (Mwambu, 1973). At the time of the study, new cases of sleeping sickness were being reported in Kamuli; Mukono and Tororo districts (Figure 1) and trypanosomes from domestic animals kept in three subcounties from within each of these districts were selected for the study.

\section{Animal survey}

An animal survey was conducted in all the three study areas. Whole blood $(5 \mathrm{ml})$ was obtained from the jugular vein of cattle, sheep and goats and from the anterior vena cava in pigs (using a 1.5" x 19G needle). The blood was collected in vacuutainers containing EDTA or heparin anticoagulant. The Haematocrit centrifugation technique (HCT), wet blood film, and thin blood smears were used to examine the blood and identify the trypanosomes and blood with T. brucei sl was inoculated in mice.

\section{Isolation of $T$. brucei sl in mice}

All blood identified by microscopy to be infected with $T$. brucei sl was inoculated in the outbred White Swiss mice. These were thereafter monitored for the development of parasitaemia, starting at day one post infection until development of parasitaemia or 60 days post infection/blood inoculation. At the peak of the first rising parasitaemia, the mice were bled and the trypanosomes were preserved in whole blood (heparin anticoagulant) with glycerol and stored in liquid nitrogen (stabilates) until needed for further characterization.

Mice inoculated with trypanosomes considered to be $T$. brucei sl and failed to developed parasitaemia after 60 days were euthanized and destroyed by incineration.

\section{Selection of the SRA-PCR as the gold standard when compared with the BIIT}

This was measured based on the accuracy of the SRA-PCR technique which has been demonstrated in the University of 
Edinburgh laboratory on $70 \mathrm{~T}$. brucei sl laboratory strains of known human infectivity status (Welburn et al., 2001). These Trypanozoons subspecies were identified through an array of tests including the BIIT, isoenzyme analysis and RFLP (Hide et al., 1994), and later by MGE-PCR (Tilley et al., 2003). To date additional studies have also demonstrated the conserved nature of SRA by the identification of this gene in stocks of a human origin (Radwanska et al., 2002; Njiru et al., 2004).

A total of 11 stabilates of $T . \quad b$. rhodesiense were included as controls and these were raised from those earlier tested and known to be so by both SRA-PCR and BIIT.

\section{Human serum incubation infectivity tests using BIIT}

The trypanosomes were grown in white Swiss mice. When parasitaemia reached $1 \mathrm{x}$ $10^{7}$ trypanosomes per $\mathrm{ml}$ of blood, as estimated by counting the parasites under a haemocytometer counting chamber, the mice were anaesthetised and bled by cardiac puncture. This heparinised blood was then combined with $1 \mathrm{ml}$ of normal human serum, collected from healthy adults and used while fresh or within 14 days after collection with storage at $-20^{\circ} \mathrm{C}$, to make a suspension of $2 \mathrm{x}$ $10^{6}$ parasites per $\mathrm{ml}$. The mixture was transferred to trypanosome culture plates (Corning Glassworks, NY, USA) and incubated at $37^{\circ} \mathrm{C}$ for 5 hours with shaking at 30 minute intervals.

The original blood incubation infectivity test has been criticised for its level of inaccuracy for many years as there are clearly a number of factors in serum that can result in variations in the results of the BIIT (Seed et al., 1990). As such, the control serum to determine the survival of the parasite away from human serum was chosen as horse, as these animals are known to be particularly susceptible to T. brucei sl and it was assumed that their serum would have no or little adverse affects on the parasites (Kihurani et al., 1994). Controls were set up by making trypanosomes suspensions in $20 \%$ heat inactivated horse serum in PSG (6:4) under similar conditions as the test samples.

Each trypanosome stock was then inoculated into a set of 10 male mice $(5$ mice for the human sera incubate and 5 mice for the control incubates). Each mouse was inoculated with $0.2 \mathrm{ml}$ of the mixture and labelled Group A (test samples) and Group B (control samples) and thereafter monitored for development of parasitaemia (breakthrough infection) or for at least 60 days. Parasites that flourished within the Group A mice were identified as potentially human infective, while trypanosomes that failed to prosper within Group A mice where designated nonhuman infective.

\section{Polymerase chain reaction \\ Preparation of genomic materials for transport}

Capillary stabilates were thawed at room temperature and the contents of a single straw spotted directly onto a Whatman FTA card (Whatman BioScience) (Picozzi et al., 2002). Cards were allowed to dry thoroughly prior to storage at $4{ }^{\circ} \mathrm{C}$ in the presence of desiccant.

\section{Preparation of materials for PCR}

FTA $2 \mathrm{~mm}$ diameter discs were prepared according to the manufacturer's instructions, i.e. $2 \times 15$ minutes wash with FTA wash reagent, followed by $2 \times 15$ minutes washes with TE buffer. The discs were then separated and placed directly into $0.2 \mathrm{ml}$ PCR tubes to air dry.

\section{Detection of Trypanozoon material within the disc}

To ensure that each disc contained parasitic material a simple reaction was carried out using the generic Trypanozoon primers (Moser et al., 1989). Briefly each 25 $\mu 1$ reaction contained $1 \mathrm{x} \mathrm{NH}_{4}$ Reaction Buffer (Bioline), $0.2 \mathrm{mM}$ dNTP, $0.4 \mu \mathrm{M}$ of each primer, $1.5 \mathrm{mM} \mathrm{MgCl}_{2}$ and 1 Unit of BIOTAQ (Bioline); amplification conditions are described in Picozzi et al. (2002).

\section{Detection of SRA gene}

The SRA gene was detected by a 35 cycle multiplex reaction (Picozzi et al., 2005). 
Each PCR included both positive and negative controls of known stocks of $T . b$. brucei, T. b. gambiense and T. b. rhodesiense prepared from FTA cards and water.

\section{Analysis of PCR products}

PCR products were separated by agarose $(1.5 \%)$ electrophoresis containing Ethidium bromide $(0.2 \mu \mathrm{g} / \mathrm{ml})$ and then visualised on a UV transilluminator.

\section{Statistical analysis}

The sensitivity and specificity of the BIIT test were calculated with the PCR results as the reference "gold" standard. Positive and negative predictive values were calculated as the proportion of true-positive results among all positively reacting samples and as the proportion of true-negative results among all negatively reacting samples respectively. The concordance between the PCR and the BIIT data was assessed by comparison of the number of positives using each technique, and determination of the Kappa ( $\kappa)$ measure of agreement (Landis \& Koch, 1977).

\section{RESULTS}

\section{Animal survey results}

A total of 4754 animals from the 3 endemic sleeping sickness zones of SE Uganda were screened for the presence of Trypanozoon parasites by microscopy. In total $365 / 4754(7.7 \%)$ of the animals were identified as infected with $T$. brucei sl. Infected blood samples were inoculated into mice to allow further characterisation of the parasitic sub-species. A total of 189/365 (51.8\%) of the T. brucei sl stocks were found to prosper within the mice. Of these, 98 samples were further analysed by the BIIT and the SRA-PCR as they had shown ability to give detectable parasitaemia within 3-6 days after mice inoculation.

\section{Blood incubation infectivity test on animal stabilates}

Being human serum resistant, a total of 43/98 stabilates were identified as being human serum resistance and as such were determined as being $T$. b. rhodesiense, this value does not include the previously mentioned $11 \quad T . \quad b$. rhodesiense control stabilates. A further 55/98 Trypanozoons stabilates were unable to survive in human serum but were still infective to mice after incubation in horse serum. These samples were determined to be T. b. brucei (Table 1).

\section{PCR analysis for presence of Trypanozoon DNA within preparations}

All 98 T. brucei sl samples from animals were found to contain detectable amounts of Trypanozoon DNA, and these samples were included in further analysis.

\section{SRA-PCR}

All samples were shown by the multiplexed PCR to contain sufficient material for the detection of a single copy gene. The differentiation between human and non-human infective parasites was therefore due to the additional presence or absence of the SRA band. A total of 39 stabilates were shown to contain the SRA gene, identifying them as $T . \quad b$. rhodesiense. The absence of this gene within the remaining 59 samples identified them as T. b. brucei (Table 1).

\section{Comparison of results}

The agreement between the BIIT and the detection of the SRA gene by PCR was $81.6 \%$ (80/98) (see Table 1). Taking the detection of the SRA gene as the gold standard, both the sensitivity (32/39) and specificity (48/59) of BIIT were $82.1 \%$ and $81.4 \%$ respectively.

The positive and negative predictive values for the BIIT test were $74.4 \%$ and $87.3 \%$ respectively. Cohen's kappa statistic $\kappa$, which gives a measure of agreement between the two tests was $\kappa=0.63$ that may be interpreted as BIIT having a substantial agreement with SRA-PCR (Landis and Koch, 1977). There was no significant difference between the two tests $\left(\chi^{2}=0.33, \mathrm{p} \leq 1, \mathrm{~d} f=1\right)$. 

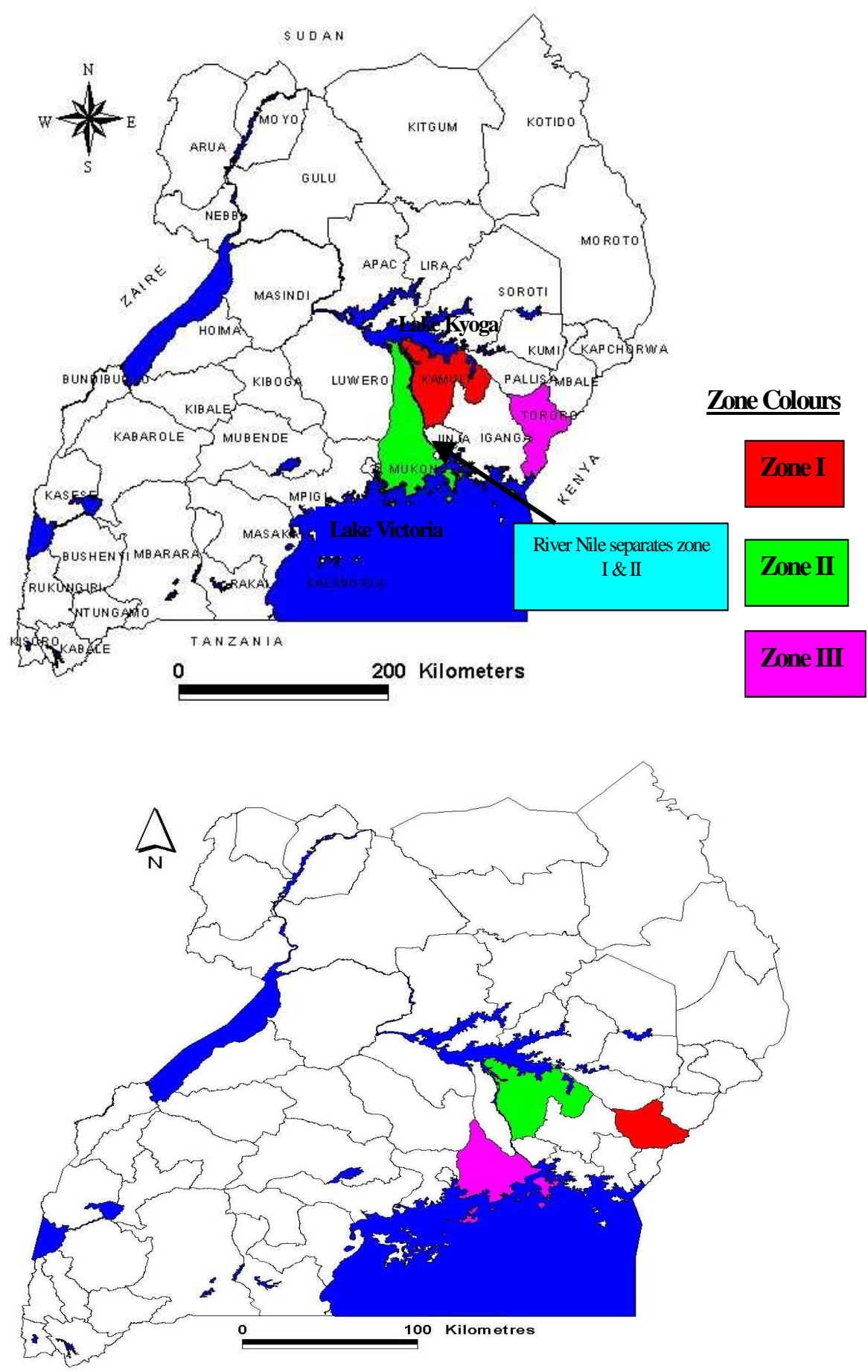

Figure 1: Map of Uganda, sites of sample collection highlighted in pink, green and red for Mukono, Kamuli and Tororo districts respectively.

Zone I (Kamuli district): One of the sleeping sickness endemic areas of Uganda

Zone II (Mukono district): One of the sleeping sickness endemic areas of Uganda Zone III (Tororo district): One of the sleeping sickness endemic areas of Uganda 
Table 1: Test results for the Blood incubation infectivity test (BIIT) and serum resistance associated gene polymerase chain reaction (SRA-PCR) on T. brucei sl. from domestic animals

\begin{tabular}{ccccc}
\hline & & \multicolumn{4}{c}{ Detection of the SRA gene } \\
\cline { 3 - 6 } & & + & - & Total \\
& + & 32 & 11 & $\mathbf{4 3}$ \\
& - & 7 & 48 & $\mathbf{5 5}$ \\
\cline { 2 - 5 } & Total & $\mathbf{3 9}$ & $\mathbf{5 9}$ & $\mathbf{9 8}$ \\
\hline
\end{tabular}

Note: This assumed SRA-PCR as the reference standard.

\section{Discussion}

Historically the identification of human infective parasites from within the animal reservoir was to confirm infectivity to human volunteers as exemplified by the identification of $T$. $b$. rhodesiense in Bushbuck (Heisch et al., 1958). Time has thank-fully moved on, so that with the advent of the BIIT test (Rickman \& Robson, 1970a, 1970b), the survival of Trypanozoons within human serum could be studied in-vitro. Similarly, the advent of molecular biology introduced a range of other applications, for example iso-enzyme analysis and RFLP (Hide et al., 1990), although these techniques require expansion of stocks to provide sufficient material for analysis and as such cannot be carried out directly on infected blood collected in the field. The development of PCR and later identification of SRA as a molecular marker for $T$. $b$. rhodesiense has lessened the requirement of high levels of starting materials, so that PCR can now be carried out directly on field samples (Welburn et al., 2001). However, the major challenge to date is that PCR based technologies for the differentiation of potentially human infective trypanosomes are not freely available across many parts of Africa (personal observation) and the BIIT is still used to determine human infectivity of $T$. brucei sl. With this study, the largest comparison into the accuracy of PCR compared with resistance to human serum showed a $100 \%$ agreement between the 32 samples tested and this study provides the largest example to date of the application of the BIIT and SRA-PCR on field samples.
Important to note is that although there was a $19 \%$ disagreement, there was no significant difference between the two tests $\left(\chi^{2}=0.33\right.$, $\mathrm{p} \leq 1, \mathrm{~d} f=1)$.

Although the difference between these tests has been observed to be minor in this study, it has been noted that in BIIT, trypanosomes must first and foremost have the ability to prosper upon infection into a mouse. The animals in this experiment were not immuno-compromised prior to inoculations and as such may have proved to be a more resilient host. Therefore, while growth of the human serum incubated trypanosomes in mice was a strong indicator of human infectivity, non-growth can not lead to classification of the $T$. brucei sl as $T$. $b$. brucei which could account for the negative predictive value of $87.3 \%$. The survival of $T$. $b$. brucei in human serum is less easy to explain and although the BIIT can easily be used in many laboratory settings, it is important to confirm the identity of the parasite as T. $b$. rhodesiense using SRA-PCR. In addition, there is a T.b. brucei reference strain (TREU927/4) that was recently observed as partially resistant to human serum and yet the SRA gene is not detectable by PCR (Radwanska et al., 2002; Turner, 2004; Vanhamme, 2004). A number of genes with homology to SRA have been identifiable which are unable to confer resistance to human serum (Turner, 2004; Vanhamme, 2004), the ease to apply SRA-PCR and short time taken to define the risk of the animal 
reservoir makes it become a technique of choice.

In conclusion, the findings suggest that although easy and relatively cheap to set up, the BIIT test alone is not sufficiently sensitive to reliably determine the potential human infectivity status of animal trypanosomes relative to molecular identification by SRAPCR. In addition, the waiting time which may sometimes require 60 days is too long and in areas with the danger of the spread of the zoonotic $T . b$. rhodesiense use of SRA-PCR is recommended as the samples to be analysed by the SRA-PCR can be collected onto FTA cards which makes the need to obtain viable stabilates of the parasites no longer a necessity.

\section{ACKNOWLEDGEMENTS}

This work was carried out with financial support from Cunningham trust, WHO/TDR/RCS and Makerere University, school of graduate studies

\section{REFERENCES}

Fairbairn M. 1933. The action of human serum in vitro on sixty-four recently isolated strains of $T$. rhodesiense. Annals of Tropical Medicine and Parasitology, 27: 185-205.

Hawking F. 1978. The resistance of Trypanosoma congolense, T.vivax and $T$. evansi to human plasma. Transactions of the Royal Society of Tropical Medicine and Hygiene, 72(4): 405-407.

Heisch RB, McMahon JP, Mansonbahr PE. 1958. The isolation of Trypanosoma rhodesiense from a bushbuck. British Medical Journal, 2(5106): 1203-1204.

Hide G, Cattand P, Le Ray D, Barry JD, Tait A. 1990. The identification of Trypanosoma brucei Sub-species using repetitive DNA sequences. Molecular and Biochemical Parasitology, 39(2): 213-225.

Hide G, Tait A, Maudlin I, Welburn SC. 1994. Epidemiological relationships of Trypanosoma brucei stocks from South East Uganda: evidence for different population structures in human infective and non-human infective isolates. Parasitology, 109(1): 95-111.

Kihurani DO, Nantulya VM, Mbiuki SM, Mogoa E, Nguhiu-Mwangi J, Mbithi PM. 1994. Trypanosoma brucei, $T$. congolense and $T$. vivax infections in horses on a farm in Kenya. Trop Anim Health Prod., 26(2): 95-101.

Landis JR. Koch GG. 1977. The measurement of observer agreement for categorical data. Biometrics., 33(1): 159-74.

Mwambu PM. 1973. Importance of cattle as natural reservoir hosts of $\mathrm{T}$. rhodesiense and resistance to ethidium (homidium bromide) of $\mathrm{T}$. brucei subgroup organisms isolated from cattle. Trans $R$ Soc Trop Med Hyg., 67(2): 286-7.

Njiru ZK, Ndung'u K, Matete G, Ndungu JM, Gibson WC. 2004. Detection of Trypanosoma brucei rhodesiense in animals from sleeping sickness foci in East Africa using the serum resistance associated (SRA) gene. Acta Tropica., 90(3): 249-54.

Picozzi K, Tilley A, F'evre EM, Coleman PG, Magona JW, Odiit M, Eisler MC, Welburn SC. 2002. The diagnosis of trypanosome infections: applications of novel technology for reducing disease risk. Afr. J. Biotechnol., 1(2): 39-45.

Picozzi K, Fevre EM, Odiit M, Carrington M, Eisler MC, Maudlin I, Welburn SC. 2005. Sleeping sickness in Uganda: a thin line between two fatal diseases. BMJ., 331: 1238-1241.

Radwanska M, Chamekh M, Vanhamme L, Claes F, Magez S, Magnus E, de Baetselier P, Buscher P, Pays E. 2002. The serum resistance-associated gene as a diagnostic tool for the detection of Trypanosoma brucei rhodesiense. Am J Trop Med Hyg., 67(6): 684-90.

Rickman L, Kolala F. 1982. Effects of some African game animal sera on Trypanosoma brucei rhodesiense and $T$. b. brucei clones. Tropenmed Parasitol. 33(2): 129-35. 
Rickman LR, Robson J. 1970a. The blood incubation infectivity test: a simple test which may serve to distinguish Trypanosoma $b$. brucei from $T . \quad b$. rhodesiense. Bull World Health Organ. 42(4): 650-1.

Rickman LR, Robson J. 1970b. The testing of proven Trypanosoma b. brucei and $T$. rhodesiense strains by the blood incubation infectivity test. Bull World Health Organ., 42(6): 911-6.

Rickman LR, Ernest A, Kanyangala S, Kunda E. 1991. Human serum sensitivities of trypanozoon isolates from naturally infected hosts in the Luangwa Valley, Zambia. East African Medical Journal, 68(11): 880-892.

Seed JR, Sechelski JB, Loomis MR 1990. A survey for a trypanocidal factor in primate sera. J Protozool. 37(5): 393400.

Tilley A, Welburn SC, Fe`vre EM Feil EJ, Hide G. 2003. Trypanosoma brucei: trypanosome strain typing using PCR analysis of mobile genetic elements (MGE-PCR). Exp Parasitol., 104(1-2): 26-32.

Turner CM, McLellan S, Lindergard LA, Bisoni L, Tait A, MacLeaod A. 2004. Human infectivity trait in Trypanosoma brucei: stability, heritability and relationship to sra expression. Parasitology, 129(4): 445-54.

Waiswa C, Olaho-Mukani W, KatungukaRwakishaya E. 2003. Domestic animals as reservoirs for sleeping sickness in three endemic foci in south-eastern Uganda. Ann Trop Med Parasitol., 97(2): 149-55.

Welburn SC, Picozzi K, Fevre EM, Coleman PG, Odiit M, Carrington M, Maudlin I. 2001. Identification of human-infective trypanosomes in animal reservoir of sleeping sickness in Uganda by means of serum-resistance-associated (SRA) gene. Lancet, 358(9298): 2017-2019.

Xong HV. 1998. A VSG expression siteassociated gene confers resistance to human serum in Trypanosoma rhodesiense. Cell, 95(6): 839-846.

Vanhamme L. 2004. The Trypanosoma brucei reference strain TREU927/4 contains T. brucei rhodesiense-specific SRA sequences, but displays a distinct phenotype of relative resistance to human serum. Mol. Biochem. Parasitol., 135(1): $39-47$. 